\title{
Coffee intake and decreased amyloid pathology in human brain
}

Jee Wook Kim ${ }^{1,2}$, Min Soo Byun ${ }^{3}$, Dahyun $\mathrm{Y}^{3}$, Jun Ho Lee ${ }^{4}$, So Yeon Jeon ${ }^{4}$, Gijung Jung ${ }^{4}$, Han Na Lee ${ }^{4}$, Bo Kyung Sohn ${ }^{5}$, Jun-Young Lee $\mathbb{1}^{6,7}$, Yu Kyeong Kim ${ }^{8}$, Seong A Shin ${ }^{8}$, Chul-Ho Sohn ${ }^{9}$ and Dong Young Lee ${ }^{3,4,7}$, for the KBASE Research Group

\begin{abstract}
Several epidemiological and preclinical studies supported the protective effect of coffee on Alzheimer's disease (AD). However, it is still unknown whether coffee is specifically related with reduced brain AD pathologies in human. Hence, this study aims to investigate relationships between coffee intake and in vivo AD pathologies, including cerebral betaamyloid (AB) deposition, the neurodegeneration of AD-signature regions, and cerebral white matter hyperintensities (WMH). A total of 411 non-demented older adults were included. Participants underwent comprehensive clinical assessment and multimodal neuroimaging including $\left[{ }^{11} \mathrm{C}\right]$ Pittsburgh compound B-positron emission tomography $(\mathrm{PET}),\left[{ }^{18} \mathrm{~F}\right]$ fluorodeoxyglucose PET, and magnetic resonance imaging scans. Lifetime and current coffee intake were categorized as follows: no coffee or $<2$ cups/day (reference category) and $\geq 2$ cups/day (higher coffee intake). Lifetime coffee intake of $\geq 2$ cups/day was significantly associated with a lower $A \beta$ positivity compared to coffee intake of $<2$ cups/day, even after controlling for potential confounders. In contrast, neither lifetime nor current coffee intake was not related to hypometabolism, atrophy of AD-signature region, and WMH volume. The findings suggest that higher lifetime coffee intake may contribute to lowering the risk of $A D$ or related cognitive decline by reducing pathological cerebral amyloid deposition.
\end{abstract}

\section{Introduction}

Coffee is one of the most popularly consumed beverages in the world and a high proportion of adults drink coffee daily ${ }^{1}$. Coffee contains hundreds of bioactive compounds, including caffeine, chlorogenic acid, polyphenols, and small amounts of minerals and vitamins, some of which are known to have positive effects on health ${ }^{2}$. Many epidemiological studies suggest that coffee has beneficial effects on various medical conditions, including stroke ${ }^{3}$, heart failure ${ }^{4}$, cancers $^{5}$, diabetes ${ }^{6}$, suicide ${ }^{7}$, Parkinson's disease $^{8}$, and mortality?

\footnotetext{
Correspondence: Dong Young Lee (selfpsy@snu.ac.kr)

'Department of Neuropsychiatry, Hallym University Dongtan Sacred Heart Hospital, 7 Keunjaebong-Gil, Hwaseong, Gyeonggi 18450, Republic of Korea ${ }^{2}$ Department of Psychiatry, Hallym University College of Medicine, Chuncheon, Gangwon 24252, Republic of Korea

Full list of author information is available at the end of the article

The coinvestigators of the KBASE Research Group are listed in elsewhere

(http://kbase.kr).
}

Several epidemiological studies also supported the protective effect of coffee on Alzheimer's disease (AD) ${ }^{10-12}$ and cognitive decline ${ }^{13-15}$. Nevertheless, there is limited information available on the neuropathological evidences that support the protective effects of coffee on AD and related cognitive decline in humans. Although a preclinical study of aged transgenic AD mice reported that caffeine, a major component of coffee, decreases brain beta-amyloid $(A \beta)$ levels ${ }^{16-18}$, it is still unknown whether coffee is specifically related with reduced brain $A D$ pathologies, including $A \beta$ deposition and regional neurodegenerations in human.

Therefore, we investigate relationships between coffee intake and in vivo $\mathrm{AD}$ biomarkers on multimodal brain imaging, including cerebral $\mathrm{A} \beta$ deposition, $\mathrm{AD}$-signature region cerebral glucose metabolism (AD-CM), ADsignature region cortical thickness (AD-CT), and

\section{(c) The Author(s) 2019}

(c) (i) Open Access This article is licensed under a Creative Commons Attribution 4.0 International License, which permits use, sharing, adaptation, distribution and reproduction cc) in any medium or format, as long as you give appropriate credit to the original author(s) and the source, provide a link to the Creative Commons license, and indicate if changes were made. The images or other third party material in this article are included in the article's Creative Commons license, unless indicated otherwise in a credit line to the material. If material is not included in the article's Creative Commons license and your intended use is not permitted by statutory regulation or exceeds the permitted use, you will need to obtain permission directly from the copyright holder. To view a copy of this license, visit http://creativecommons.org/licenses/by/4.0/. 
cerebral white matter hyperintensities (WMH) in nondemented older adults.

\section{Methods \\ Participants}

This study was part of the Korean Brain Aging Study for Early Diagnosis and Prediction of Alzheimer's Disease (KBASE), which is an ongoing prospective cohort study that begun in $2014^{19}$. As of February 2017, 411 individuals [282 cognitively normal $(\mathrm{CN})$ adults, and 129 adults with mild cognitive impairment (MCI)], between 55 and 90 years of age were enrolled in the study.

The CN group consisted of participants with a Clinical Dementia Rating $(\mathrm{CDR})^{20}$ score of 0 and no diagnosis of MCI or dementia. All participants with MCI met the current consensus criteria for amnestic MCI, including: (1) memory complaints confirmed by an informant; (2) objective memory impairments; (3) preservation of global cognitive function; (4) independence in functional activities; and (5) no dementia. Regarding Criterion 2, the age-, education-, and gender-adjusted $z$-score was $<-1.0$ for at least one of four episodic memory tests: Word List Memory, Word List Recall, Word List Recognition, and Constructional Recall tests; these are included in the Korean version of the Consortium to Establish a Registry for Alzheimer's Disease (CERAD-K) neuropsychological battery $^{21}$. All MCI individuals had a CDR score of 0.5. The exclusion criteria were as follows: (1) presence of a major psychiatric illness; (2) significant neurological or medical condition or comorbidity that could affect mental functioning; (3) contraindications for an magnetic resonance imaging (MRI) scan (e.g., pacemaker or claustrophobia); (4) illiteracy; (5) the presence of significant visual/hearing difficulties and/or severe communication or behavioral problems that would make clinical examinations or brain scans difficult; (6) pregnant or lactation; (7) use of an investigational drug; and (8) drinking tea extract regularly. The Institutional Review Board of Seoul National University Hospital and the SMG-SNU Boramae Medical Center in South Korea approved the present study, and all subjects provided written informed consent prior to participation. More detailed information on recruitment of the KBASE cohort is described in our previous report ${ }^{19}$.

\section{Clinical and neuropsychological assessments}

All participants were administered standardized clinical assessments by trained board-certified psychiatrists based on the KBASE clinical assessment protocol which incorporated the CERAD-K clinical assessment ${ }^{19}$, which incorporates the CERAD- $\mathrm{K}^{22}$. All subjects were also given a comprehensive neuropsychological assessment battery, administered by a clinical neuropsychologist or trained psychometrists according to a standardized protocol incorporating the CERAD-K neuropsychological battery ${ }^{21}$. Details on full assessment battery were described previously ${ }^{19}$.

\section{Assessment of coffee intake}

All participants were systematically assessed by trained nurses to determine coffee intake. Specifically, the amount of coffee intake (cups/day) for each participant were assessed for the past one year (i.e., current) and overall lifetime. Previous epidemiologic studies on the effect of coffee intake ${ }^{10,12,23}$ showed that there was a clear difference in the risk of overall or AD dementia between " $<2$ cups/day (no or lower drinker)" and " $\geq 2$ cups/day (higher drinker)" group. Based on the findings, we categorized the participant into the two group, and tried to test the hypothesis that there is a difference in $\mathrm{AD}$ pathology between the two.

\section{Assessment of potential confounders}

Coffee intake may be influenced by various other conditions. Therefore, all participants were systematically evaluated about potential confounders, such as lifetime cognitive activity (LCA), occupational complexity, annual income, vascular risk, depression, smoking, and alcohol intake.

Cognitive activity participation frequency was measured by 39 -item structured questionnaires ${ }^{24,25}$. The details of the measurement of cognitive activity are described in our previous report ${ }^{26}$. Item scores were averaged to yield separate values for each age period. We then calculated the composite score of LCA to use in the subsequent analysis which was an average of all 4-epoch means. With regard to occupational complexity, we considered only the longest-held occupation and then classified into four levels based on the skill levels described in International Standard Classification of Occupations (http://www.ilo. org/public/english/bureau/stat/isco/). Occupations typically involve simple and routine physical or manual tasks at skill level 1, the performance of tasks, such as operating machinery and electronic equipment; driving vehicles; maintenance and repair of electrical and mechanical equipment; and manipulation, ordering and storage of information at skill level 2, the performance of complex technical and practical tasks that require complex problem solving, reasoning, and decision making in a specialized field at skill level 3, and the performance of tasks that require complex problem-solving, decision-making, and creativity based on an extensive body of theoretical and factual knowledge in a specialized field at skill level 4 . Information about occupation was obtained from selfreport by the participants and confirmed by reliable informants. Annual income was evaluated and categorized into three groups (below the minimum cost of living (MCL), more than MCL but below twice the MCL, twice the MCL or more (http://www.law.go.kr). The MCL was 
determined according to the administrative rule published by the Ministry of Health and Welfare, Republic of Korea in November 2012. The MCL was 572,168 Korea Won (KRW) for single-person household and added 286,840 KRW for each additional housemate. The comorbidity rates of vascular risk factors were assessed by interviews of participants and their reliable informants; a vascular risk score (VRS) was calculated based on the number of vascular risk factors present and reported as a percentage ${ }^{27}$. To acquire accurate information, reliable informants were interviewed, and medical records were reviewed. The Geriatric Depression Scale (GDS) ${ }^{28}$ was used to measure the severity of depressive symptoms. Smoking status (never/former/smoker) and alcohol intake status (never/ former/drinker) were evaluated through nurse interview. Blood samples were also obtained via venipuncture, genomic DNA was extracted from whole blood and apolipoprotein E (APOE) genotyping was performed as described previously ${ }^{29}$. APOE $\varepsilon 4$ (APOE4) positivity was defined as the presence of at least one $\varepsilon 4$ allele was present.

\section{Measurement of cerebral $A \beta$ deposition}

All participants underwent simultaneous threedimensional $\left[{ }^{11} \mathrm{C}\right]$ Pittsburg compound B (PiB)-positron emission tomography (PET) and T1-weighted MRI scans using a $3.0 \mathrm{~T}$ Biograph mMR (PET-MR) scanner (Siemens; Washington DC, WC, USA) according to the manufacturer's guidelines. The details of PiB-PET acquisition and preprocessing were described in our previous report $^{30}$. An AAL algorithm and a region-combining method $^{31}$ were applied to determine the regions of interest (ROIs) for characterization of $\mathrm{PiB}$ retention levels in the frontal, lateral parietal, posterior cingulate-precuneus, and lateral temporal regions. The standardized uptake value ratio (SUVR) values for each ROI were calculated by dividing the mean value for all voxels within each ROI by the mean cerebellar uptake value on the same image. Each participant was classified as $A \beta$ positive $(\mathrm{A} \beta+)$ if the SUVR value was $>1.4$ in at least one of the four ROIs ${ }^{31,32}$. Considering the bimodal distribution of our $\mathrm{PiB}$ data, only $\mathrm{A} \beta$ positivity was used as an outcome variable $^{33,34}$.

\section{Measurement of AD-CM}

All subjects underwent $\left[{ }^{18} \mathrm{~F}\right]$ fluorodeoxyglucose (FDG)PET imaging using the above-described PET-MR machine. The details of FDG-PET acquisition and preprocessing were described in our previous report ${ }^{30}$. ADsignature FDG ROIs that are sensitive to the changes associated with $\mathrm{AD}$, such as the angular gyri, posterior cingulate cortex, and inferior temporal gyri ${ }^{32}$, were determined. AD-CM was defined as the voxel-weighted mean SUVR extracted from the AD-signature FDG ROIs.

\section{Measurement of AD-CT}

All T1-weighted images were acquired in the sagittal orientation using the above-described 3.0 T PET-MR machine. MR image acquisition and preprocessing were described in our previous report ${ }^{30}$. AD-CT was defined as the mean cortical thickness values obtained from $\mathrm{AD}$ signature regions including the entorhinal, inferior temporal, middle temporal, and fusiform gyrus, as described previously $^{32}$.

\section{Measurement of WMH}

All participants underwent MRI scans with fluid attenuated inversion recovery using the abovementioned 3.0 T PET-MR scanner in a validated automatic procedure that has previously been reported ${ }^{35}$. The details of the volume measurement of cerebral WMH were previously described $^{36}$.

\section{Statistical analysis}

We first compared demographic variables, other potential confounders [APOE4, clinical diagnosis (CN vs. $\mathrm{MCI}$ ), LCA score, occupational complexity, annual income status, VRS, GDS score, smoking status, and alcohol intake status] for the relationship between coffee intake and $\mathrm{AD}$ biomarkers, and $\mathrm{AD}$ imaging biomarkers between lifetime coffee intake categories ( $<2$ cups/day and $\geq 2$ cups/day) by $t$ test or $\chi^{2}$ test as appropriate. In order to explore the relationship between lifetime coffee intake amount and potential confounders, we performed Spearman correlation analyses. To examine the relationships between lifetime (or current) coffee intake category and neuroimaging parameters, multivariate logistic or linear regression analyses were performed as appropriate. In these analyses, " $<2$ cups/day" category was used as a reference. Three models were tested for controlling the covariates stepwisely. The first model included age, gender, education, APOE4, clinical diagnosis as covariates; the second model included covariates in the first model plus LCA score, occupational complexity, annual income status, VRS, GDS score, smoking status, and alcohol intake status; and third model included covariates in the second model plus the duration of coffee intake and the age of first coffee intake. To reduce false positive error due to multiple testing, we applied Bonferroni correction. Actually, $p<0.00625(=0.05 / 8)$ was used as the threshold for statistical significance for each analysis considering 4 biomarkers and 2 time periods.

For the AD neuroimaging biomarker with significant association with coffee intake in above analyses, additional exploratory analyses were performed. First, to explore whether there are any brain regional specificity in regard of the relationship between lifetime coffee intake and the biomarker, the same analysis was done for each of the four ROI (i.e., the frontal, lateral parietal, posterior 
cingulate-precuneus, and lateral temporal region). Second, in order to investigate the modulating effects of the potential confounders (i.e., age, gender, education, APOE4, clinical diagnosis, LCA score, occupational complexity, annual income status, VRS, GDS score, smoking status, and alcohol intake status) on the relationships between coffee intake and the biomarker, we performed the same analysis including two-way interaction term between coffee intake and any one of the confounders, as well as coffee intake itself, as an independent variable. We additionally examined the three-way interaction between lifetime coffee intake and any two of age, education, gender, and APOE4 on the relationship between coffee intake and the biomarker. Third, to explore the dose-effect relationship between overall amount of coffee intake and the biomarker, the same analysis including the total amount of lifetime coffee intake (=duration of coffee intake $\times$ cups of coffee intake/ day) as an independent variable instead of coffee intake category (lower vs. higher) were performed. For similar purpose, we also compared the AD biomarker among four coffee intake categories (i.e., 0 or $<1$ cups/day, $1 \leq$ and $<2$ cups/day, $2 \leq$ and $<3$ cups/day, and $3 \leq$ cups/day) instead of the dichotomous categories by using $\chi^{2}$ test. For these exploratory analyses, $p<0.05$ was served as a statistical threshold. All statistical analyses were performed using IBM SPSS Statistics 24 software (IBM Corp., Armonk, NY, USA).

\section{Results}

\section{Participant characteristics}

The demographic and clinical characteristics of the participants are presented by the categories of lifetime coffee intake in Table 1 . Of the 411 participants, 269 were no or lower coffee drinkers ( $<2$ cups/day) and 142 were higher coffee drinkers ( $\geq 2$ cups/day). There were significant differences of sex, education, duration of coffee intake, age of first coffee intake, LCA score, occupational complexity, smoking status, alcohol drinking status, and $A \beta$ positivity between the two lifetime coffee intake groups. Correlations of lifetime coffee intake amount with potential confounders for the relationship between coffee intake and AD biomarkers were also presented in Supplementary Table 1.

\section{Difference of $A \beta$ positivity between high and low coffee intakes}

The association between coffee intake and $A \beta$ positivity presented in Table 2 and Fig. 1. Lifetime coffee intake of $\geq 2$ cups/day showed significantly lower $A \beta$ positivity compared to coffee intake of $<2$ cups/day, regardless of the models. To explore whether there are any brain regional specificity in regard of the relationship between lifetime coffee intake and $A \beta$ positivity, the difference of
$\mathrm{A} \beta$ positivity between high and low lifetime coffee intakes was tested for each of the four ROI (i.e., the frontal, lateral parietal, posterior cingulate-precuneus, and lateral temporal region). Lifetime coffee intake of $\geq 2$ cups/day showed lower $A \beta$ positivity in all four regions (Table 3 ). In contrast to lifetime coffee intake, current coffee intake was not related to $A \beta$ positivity regardless of the covariates.

\section{Moderating effect of potential confounders on the relationship between lifetime coffee intake and $A \beta$ positivity}

Any two-way interaction between lifetime coffee intake and each of age, gender, gender, APOE4, clinical diagnosis, LCA score, occupational complexity, annual income status, VRS, GDS score, smoking status, and alcohol intake status was not significant, indicating that the potential confounders do not moderate the relationship between lifetime coffee intake and $A \beta$ positivity (Supplementary Table 2). We additionally examined the three-way interaction between lifetime coffee intake and any two of age, gender, education, and APOE4 on the relationship between coffee intake and $A \beta$ positivity, but did not find any significant finding.

\section{Dose-effect relationship between lifetime coffee intake and $A \beta$ positivity}

To explore the dose-effect relationship between lifetime coffee intake amount and $A \beta$ positivity further, we compared $A \beta$ positivity rates according to four lifetime coffee intake strata, i.e., 0 or $<1$ cups/day, $1 \leq$ and $<2$ cups/day, $2 \leq$ and $<3$ cups/day, and $3 \leq$ cups/day by using $X^{2}$ test. As shown in Supplementary Fig. 1, there was a significant trend of association between lifetime coffee intake strata and $\mathrm{A} \beta$ positivity $(p=0.048)$. Multiple logistic regression analysis also demonstrated that there was a trend toward significance on dose-effect association between the total amount of lifetime coffee intake (=duration of coffee intake $\times$ cups of coffee intake/day) and $A \beta$ positivity [OR $(95 \%$ CI $)=0.991 \quad(0.982-1.001), \quad p=0.067] . \quad$ As the amount increased, so A $\beta$-positivity rate decreased (Supplementary Table 3).

\section{Association of coffee intake with cerebral tau deposition, $\mathrm{AD}-\mathrm{CM}, \mathrm{AD}-\mathrm{CT}$, and WMH}

In contrast to the results for $A \beta$ positivity, neither lifetime nor current coffee intake was related with any of $A D$ CM, AD-CT, and WMH (Table 4).

\section{Discussion}

The present study found that a lifetime coffee intake of $\geq 2$ cups/day (higher coffee intake) was associated with lower cerebral $A \beta$ positivity rate in non-demented older adults when compared to the coffee intake of $<2$ cups/day. 
Table 1 Participant characteristics ${ }^{a}$

\begin{tabular}{|c|c|c|c|c|c|}
\hline \multirow[t]{2}{*}{ Characteristic } & \multicolumn{3}{|c|}{ Coffee intake amount, lifetime } & \multirow[t]{2}{*}{$t$ or $x^{2}$} & \multirow[t]{2}{*}{$p$ Value } \\
\hline & $<2$ cups/day & $\geq 2$ cups/day & Total & & \\
\hline$n$ & 269 & 142 & 411 & & \\
\hline Age, y & $71.06(7.73)$ & $69.67(8.43)$ & $70.58(8.00)$ & 1.675 & 0.095 \\
\hline Female, no. (\%) & $175(65.06)$ & $57(40.04)$ & $232(56.45)$ & 23.467 & $<0.001$ \\
\hline Education, y & $10.56(4.90)$ & $12.27(4.49)$ & $11.15(4.82)$ & -3.574 & $<0.001$ \\
\hline MMSE & $25.26(3.41)$ & $25.96(3.34)$ & $25.50(3.40)$ & -2.007 & 0.045 \\
\hline APOE4 positivity, no. (\%) & $61(22.76)$ & $35(24.65)$ & $96(23.41)$ & 0.184 & 0.668 \\
\hline Clinical diagnosis, CN, no. (\%) & $183(68.03)$ & $99(69.72)$ & $282(68.61)$ & 0.123 & 0.726 \\
\hline Duration of coffee intake, $y$ & $27.61(19.06)$ & $34.12(15.06)$ & $25.93(18.73)$ & -6.784 & $<0.001$ \\
\hline Age of first coffee intake, y & $41.17(17.94)$ & $34.03(14.54)$ & $38.31(17.01)$ & 3.940 & $<0.001$ \\
\hline \multicolumn{6}{|l|}{ Cognitive activity } \\
\hline Childhood score & $2.00(0.64)$ & $2.06(0.58)$ & $2.02(0.62)$ & -0.892 & 0.373 \\
\hline Adulthood score & $2.29(0.90)$ & $2.46(0.84)$ & $2.35(0.86)$ & -1.800 & 0.073 \\
\hline Midlife score & $2.24(0.84)$ & $2.44(0.79)$ & $2.31(0.83)$ & -2.338 & 0.020 \\
\hline Current score & $2.37(0.69)$ & $2.52(0.71)$ & $2.42(0.70)$ & -1.934 & 0.054 \\
\hline Lifetime composite score & $2.23(0.67)$ & $2.37(0.59)$ & $2.27(0.64)$ & -2.113 & 0.035 \\
\hline Occupational complexity, no. (\%) & & & & 11.571 & 0.021 \\
\hline None & $59(22.01)$ & $16(11.27)$ & $75(18.29)$ & & \\
\hline Skill level 1 & $20(7.46)$ & $9(6.34)$ & $29(7.07)$ & & \\
\hline Skill level 2 & $88(32.84)$ & $44(30.99)$ & $132(30.19)$ & & \\
\hline Skill level 3 & $28(10.45)$ & $26(18.31)$ & $54(13.17)$ & & \\
\hline Skill level 4 & $73(27.24)$ & $47(33.10)$ & $120(29.27)$ & & \\
\hline Annual income, no. (\%) & & & & 2.530 & 0.282 \\
\hline$<\mathrm{MCL}$ & $19(7.06)$ & $16(11.27)$ & $35(8.52)$ & & \\
\hline$\geq M C L,<2 \times M C L$ & $124(46.10)$ & $58(40.85)$ & $182(44.28)$ & & \\
\hline$\geq 2 \times M C L$ & $126(46.84)$ & $68(47.89)$ & $194(47.20)$ & & \\
\hline VRS & $18.77(15.76)$ & $16.31(17.47)$ & $17.92(16.39)$ & 0.148 & 0.148 \\
\hline GDS score & $6.65(5.95)$ & $6.47(6.76)$ & $6.59(6.24)$ & 0.270 & 0.787 \\
\hline Smoking status, no. (\%) & & & & 27.087 & $<0.001$ \\
\hline Never & 206 (76.58) & $73(51.41)$ & $279(67.88)$ & & \\
\hline Former & $54(20.07)$ & $58(40.85)$ & $112(27.25)$ & & \\
\hline Smoker & $9(3.35)$ & $11(7.75)$ & $20(4.87)$ & & \\
\hline Alcohol drink status, no. (\%) & & & & 12.651 & 0.002 \\
\hline Never & $161(59.85)$ & $63(44.37)$ & $224(54.50)$ & & \\
\hline Former & $25(9.29)$ & $28(19.72)$ & $53(12.90)$ & & \\
\hline Drinker & $83(30.86)$ & $51(35.92)$ & $134(32.60)$ & & \\
\hline \multicolumn{6}{|l|}{ Cerebral A $\beta$ deposition } \\
\hline$A \beta$ positivity, no. (\%) & $73(27.14)$ & $25(17.61)$ & $98(23.84)$ & 4.650 & 0.031 \\
\hline
\end{tabular}


Table 1 continued

\begin{tabular}{|c|c|c|c|c|c|}
\hline \multirow[t]{2}{*}{ Characteristic } & \multicolumn{3}{|c|}{ Coffee intake amount, lifetime } & \multirow[t]{2}{*}{$t$ or $x^{2}$} & \multirow[t]{2}{*}{$p$ Value } \\
\hline & $<2$ cups/day & $\geq 2$ cups/day & Total & & \\
\hline \multicolumn{6}{|l|}{ Neurodegeneration } \\
\hline AD-CM, SUVR & $1.40(0.13)$ & $1.39(0.12)$ & $1.39(0.13)$ & 0.462 & 0.645 \\
\hline $\mathrm{AD}-\mathrm{CT}, \mathrm{mm}$ & $2.81(0.22)$ & $2.80(0.23)$ & $2.81(0.22)$ & 0.262 & 0.794 \\
\hline WMH volume, $\mathrm{cm}^{3}$ & $5.89(5.56)$ & $6.02(5.03)$ & $5.94(5.37)$ & -0.217 & 0.828 \\
\hline
\end{tabular}

APOE4 apolipoprotein $\varepsilon 4, C N$ cognitive normal, $M C L$ minimum cost of living, $V R S$ vascular risk score, GDS Geriatric depression scale, $A \beta$ beta-amyloid, $A D$ Alzheimer's disease, $A D-C M$ Alzheimer's disease signature cerebral glucose metabolism, $A D-C T$ Alzheimer's disease signature cortical thickness, SUVR standardized uptake value ratio, $W M H$ white matter hyperintensities

aUnless otherwise indicated, data are expressed as mean (standard deviation)

Table 2 Results of multiple logistic regression analyses for assessing the relationships of stratified coffee intake with $A \beta$ positivity in non-demented individuals

\begin{tabular}{|c|c|c|}
\hline \multirow[t]{2}{*}{ Coffee intake } & \multicolumn{2}{|l|}{$A \beta$ positivity } \\
\hline & OR (95\% CI) & $p$ Value \\
\hline \multicolumn{3}{|l|}{ Model $1^{a}$} \\
\hline \multicolumn{3}{|l|}{ Lifetime } \\
\hline$<2$ cup/day & Reference & \\
\hline$\geq 2$ cup/day & 0.401 (0.208 to 0.772$)$ & $0.006^{*}$ \\
\hline \multicolumn{3}{|l|}{ Current } \\
\hline$<2$ cup/day & Reference & \\
\hline$\geq 2$ cup/day & $0.453(0.236$ to 0.869$)$ & 0.017 \\
\hline \multicolumn{3}{|l|}{ Model $2^{b}$} \\
\hline \multicolumn{3}{|l|}{ Lifetime } \\
\hline$<2$ cup/day & Reference & \\
\hline$\geq 2$ cup/day & $0.386(0.197$ to 0.757$)$ & $0.006^{*}$ \\
\hline \multicolumn{3}{|l|}{ Current } \\
\hline$<2$ cup/day & Reference & \\
\hline$\geq 2$ cup/day & $0.443(0.227$ to 0.862$)$ & 0.017 \\
\hline \multicolumn{3}{|l|}{ Model $3^{c}$} \\
\hline \multicolumn{3}{|l|}{ Lifetime } \\
\hline$<2$ cup/day & Reference & \\
\hline$\geq 2$ cup/day & $0.334(0.162$ to 0.689$)$ & $0.003^{*}$ \\
\hline \multicolumn{3}{|l|}{ Current } \\
\hline$<2$ cup/day & Reference & \\
\hline$\geq 2$ cup/day & 0.402 (0.197 to 0.822$)$ & 0.013 \\
\hline
\end{tabular}

$A \beta$ beta-amyloid, $O R$ odds ratio, $C l$ confidence interval, APOE4 apolipoprotein $\varepsilon 4$, LCA lifetime cognitive activity, VRS vascular risk score, GDS geriatric depression scale

a Adjusted for age, gender, education, apolipoprotein $\varepsilon 4$, and clinical diagnosis

${ }^{b}$ Adjusted for covariates in Model 1 plus, LCA score, occupational complexity, and annual income status, VRS, GDS score, smoking status, and alcohol status c Adjusted for covariates in Model 2 plus, duration of coffee intake and age of first coffee intake

*Statistically significant $(p<0.00625)$

We did not find any association of coffee intake with regional neurodegeneration and WMH. This is the first study to investigate the association between higher coffee intake and in vivo $\mathrm{AD}$ pathologies in human.
The present finding of the relationship between higher coffee intake and a decreased rate of pathological $A \beta$ deposition is in line with results from previous studies using animal models, which indicated that higher caffeine, one of the major ingredients of coffee, intake exerts a protective effect via molecular $A \beta$-related mechanisms $^{16-18,37,38}$. For example, Arendash et al. ${ }^{18}$ suggested that caffeine protects $\mathrm{AD}$ mice against cognitive impairment and reduces brain $A \beta$ production by deactivating the positive-feedback loop from the $\gamma$ - to $\beta$-secretase cleavages on the $A \beta$ protein precursor. The same group also reported that high caffeine intake improves cognitive performance of aged AD mice, but not of aged wild-type mice, with reduced brain $A \beta$ levels, suggesting that the cognitive enhancing effect of caffeine in $A D$ mice is mediated by a decrease in $A \beta$ concentration ${ }^{16}$. Furthermore, Cao et al. reported that caffeine suppresses $A \beta$ levels in the plasma and brain of $A D$ mice ${ }^{17}$ and also suggested that caffeine and other components in coffee may synergize to protect against cognitive decline in $\mathrm{AD}$ mice $^{38}$. Moreover, Li et al. ${ }^{37}$ indicated that caffeine suppresses $A \beta$ protein precursor internalization and $A \beta$ generation via adenosine A3 receptor-mediated actions. The present finding also provides a neuropathological explanation for the relationship between higher coffee intake and reduced risk of $\mathrm{AD}$ dementia observed in several clinical and epidemiological studies ${ }^{10-12}$. Those studies reported higher coffee drinkers had $31-65 \%$ decrease in the risk of $\mathrm{AD}$ dementia, which is quite comparable to about $65 \%$ decrease of $\mathrm{A} \beta$ positivity rate in higher coffee drinkers (27.14\%) compared to lower coffee drinkers (17.61\%). Furthermore, the relationship between higher coffee intake and lower $A \beta$ positivity was prominent for lifetime coffee intake than for current coffee intake. This suggests that the protective effects of higher coffee intake against $A \beta$ pathology involve the chronic effects associated with prolonged exposure rather than an acute or short-term effect.

In the present study, we did not find any association of coffee intake with regional neurodegeneration and WMH. 
(A)

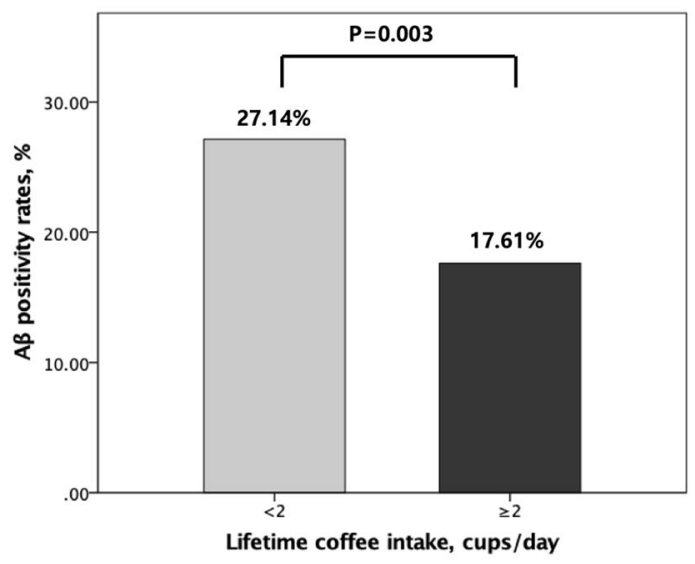

(B)

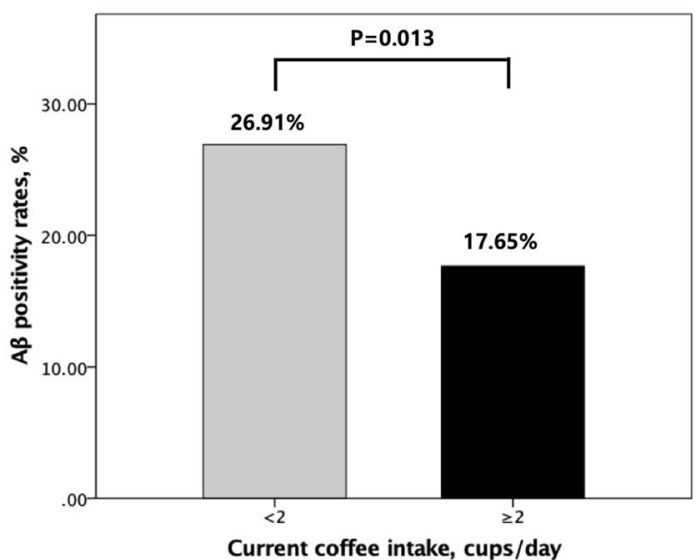

Fig. 1 A $\beta$ positivity rates according to the categories of (a) lifetime or (b) current coffee intake: comparison of A positivity rates between 0 or $<2$ cups/day and $\geq 2 \mathrm{cups} /$ day in non-demented older adults. Multivariate logistic regression analyses were performed after controlling for age, gender, education, apolipoprotein $\varepsilon 4$, clinical diagnosis, LCA score, occupational complexity, annual income status, VRS, GDS score, smoking status, alcohol intake status, duration of coffee intake and age of first coffee intake. A $\beta$ beta-amyloid, LCA lifetime cognitive activity, VRS vascular risk score, GDS geriatric depression scale. The English in this document has been checked by at least two professional editors, both native speakers of English. For a certificate, please see: http://www.textcheck.com/certificate/1uduau

Table 3 Results of multiple logistic regression analyses for assessing the relationship between stratified lifetime coffee intake and subregional $A \beta$ positivity in non-demented individuals

\begin{tabular}{|c|c|c|c|c|c|c|c|c|}
\hline \multirow[t]{2}{*}{ Lifetime coffee intake } & \multicolumn{2}{|l|}{ Frontal region } & \multicolumn{2}{|l|}{ PC-PRC region } & \multicolumn{2}{|l|}{ Lt. parietal region } & \multicolumn{2}{|l|}{ Lt. temporal region } \\
\hline & OR $(95 \% \mathrm{Cl})$ & $p$ & OR $(95 \% \mathrm{Cl})$ & $p$ & OR $(95 \% \mathrm{Cl})$ & $p$ & OR $(95 \% \mathrm{Cl})$ & $p$ \\
\hline \multicolumn{9}{|l|}{ Model $1^{\mathrm{a}}$} \\
\hline$<2$ cup/day & Reference & & Reference & & Reference & & Reference & \\
\hline$\geq 2$ cup/day & 0.400 (0.197 to 0.813$)$ & 0.011 & 0.417 (0.218 to 0.798$)$ & 0.008 & 0.393 (0.197 to 0.783 ) & 0.008 & $0.500(0.252$ to 0.993$)$ & 0.048 \\
\hline \multicolumn{9}{|l|}{ Model $2^{\mathrm{b}}$} \\
\hline$<2$ cup/day & Reference & & Reference & & Reference & & Reference & \\
\hline$\geq 2$ cup/day & 0.381 (0.183 to 0.793$)$ & 0.010 & 0.402 (0.206 to 0.783$)$ & 0.007 & 0.370 (0.181 to 0.757$)$ & 0.007 & 0.475 (0.234 to 0.966$)$ & 0.040 \\
\hline \multicolumn{9}{|l|}{ Model $3^{c}$} \\
\hline$<2$ cup/day & Reference & & Reference & & Reference & & Reference & \\
\hline$\geq 2$ cup/day & 0.324 (0.149 to 0.707$)$ & 0.005 & 0.349 (0.170 to 0.713$)$ & 0.004 & 0.285 (0.132 to 0.617$)$ & 0.001 & 0.400 (0.188 to 0.851$)$ & 0.017 \\
\hline
\end{tabular}

$A \beta$ beta-amyloid, $P C-P R C$ posterior cingulate-precuneus, $O R$ odds ratio, $C l$ confidence interval, $A P O E 4$ apolipoprotein $\varepsilon 4, L C A$ lifetime cognitive activity, $V R S$ vascular risk score, GDS geriatric depression scale

${ }^{a}$ Adjusted for age, gender, education, apolipoprotein $\varepsilon 4$, and clinical diagnosis

${ }^{b}$ Adjusted for covariates in Model 1 plus, LCA score, occupational complexity, annual income status, VRS, GDS score, smoking status, and alcohol status

${ }^{\mathrm{C}}$ Adjusted for covariates in Model 2 plus, duration of coffee intake and age of first coffee intake

Although no previous study investigated the relationship between coffee intake and brain metabolism, the Honolulu-Asia Aging Study showed that coffee intake was not associated with generalized brain atrophy and microvascular ischemic lesions ${ }^{39}$, similarly to our findings. In addition, the Health Professional Follow-up Study also showed that chronic coffee or caffeine intake is not associated with a risk of cerebrovascular or cardiovascular disease $^{40}$. Although some previous reports indicated an association between coffee intake and cerebrovascular risk, they examined the acute effect of coffee intake, but not the chronic effect of long-term coffee intake ${ }^{41,42}$. Such a null association between coffee intake and AD-related neurodegeneration or vascular changes indicates that chronic coffee intake has no direct effects on neurodegenerative or cerebrovascular changes through 
Table 4 Results of multiple linear model analyses for assessing the relationship between stratified coffee intake and ADCM, AD-CT, or WMH volume in non-demented individuals

\begin{tabular}{|c|c|c|c|c|c|c|}
\hline \multirow[t]{2}{*}{ Coffee intake } & \multicolumn{2}{|l|}{ AD-CM } & \multicolumn{2}{|l|}{$\mathrm{AD}-\mathrm{CT}$} & \multicolumn{2}{|l|}{ WMH } \\
\hline & $B(95 \% \mathrm{Cl})$ & $p$ Value & B $(95 \% \mathrm{Cl})$ & $p$ value & $B(95 \% \mathrm{Cl})$ & $p$ Value \\
\hline \multicolumn{7}{|l|}{ Model $1^{\mathrm{a}}$} \\
\hline \multicolumn{7}{|l|}{ Lifetime } \\
\hline$<2$ cup/day & Reference & & Reference & & Reference & \\
\hline$\geq 2$ cup/day & $-0.007(-0.034$ to 0.021$)$ & 0.633 & $0.002(-0.037$ to 0.042$)$ & 0.910 & $0.237(-0.990$ to 1.464$)$ & 0.704 \\
\hline \multicolumn{7}{|l|}{ Current } \\
\hline$<2$ cup/day & Reference & & Reference & & Reference & \\
\hline$\geq 2$ cup/day & $-0.009(-0.037$ to 0.019$)$ & 0.540 & $-0.001(-0.041$ to 0.040$)$ & 0.980 & $0.620(-0.626$ to 1.867$)$ & 0.328 \\
\hline \multicolumn{7}{|l|}{ Model $2^{\mathrm{b}}$} \\
\hline \multicolumn{7}{|l|}{ Lifetime } \\
\hline$<2$ cup/day & Reference & & Reference & & Reference & \\
\hline$\geq 2$ cup/day & $-0.008(-0.035$ to 0.020$)$ & 0.580 & $0.003(-0.037$ to 0.042$)$ & 0.888 & $0.282(-0.961$ to 1.526$)$ & 0.655 \\
\hline \multicolumn{7}{|l|}{ Current } \\
\hline$<2$ cup/day & Reference & & Reference & & Reference & \\
\hline$\geq 2$ cup/day & $-0.013(-0.040$ to 0.015$)$ & 0.369 & $-0.002(-0.041$ to 0.038$)$ & 0.935 & $0.674(-0.591$ to 1.939$)$ & 0.295 \\
\hline \multicolumn{7}{|l|}{ Model $3^{c}$} \\
\hline \multicolumn{7}{|l|}{ Lifetime } \\
\hline$<2$ cup/day & Reference & & Reference & & Reference & \\
\hline$\geq 2$ cup/day & $0.006(-0.022$ to 0.033$)$ & 0.678 & $0.008(-0.033$ to 0.048$)$ & 0.707 & $0.181(-1.124$ to 1.486$)$ & 0.785 \\
\hline \multicolumn{7}{|l|}{ Current } \\
\hline$<2$ cup/day & Reference & & Reference & & Reference & \\
\hline$\geq 2$ cup/day & $0.001(-0.027$ to 0.029$)$ & 0.951 & $0.004(-0.038$ to 0.045$)$ & 0.864 & $0.612(-0.714$ to 1.938$)$ & 0.365 \\
\hline
\end{tabular}

$A \beta$ beta-amyloid, $A D-C M$ Alzheimer's disease signature cerebral glucose metabolism, $A D$ - $C T$ Alzheimer's disease signature cortical thickness, WMH white matter hyperintensities, $C l$ confidence interval, LCA lifetime cognitive activity, GDS geriatric depression scale, APOE4 apolipoprotein $\varepsilon 4$

${ }^{a}$ Adjusted for age, gender, education, APOE4, and clinical diagnosis

${ }^{\mathrm{b}}$ Adjusted for covariates in Model 1 plus, LCA score, occupational complexity, annual income status, vascular risk score, GDS score, smoking status, and alcohol status

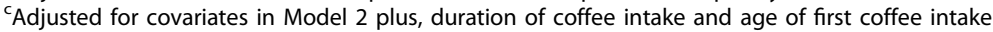

A $\beta$-independent mechanisms. Given the significant association between higher coffee intake and lower $A \beta$ positivity, the negative finding for AD-related regional neurodegeneration appears related to the long-time delay between pathological $A \beta$ accumulation and A $\beta$-dependent neurodegeneration ${ }^{43,44}$.

The present study had several limitations that should be considered. First, because this was a cross-sectional study, it is difficult to infer causal relationships from the findings. However, the significant relationship between lifetime coffee intake and amyloid pathology supports the possible causal nature of the relationship. Second, underestimates of coffee intake or retrospective recall bias may have affected the results of lifetime coffee intake in older individuals. However, coffee intake is less prone to misreporting because coffee intake is a long-term habitual behavior. Evaluation for coffee intake is known to be performed with the highest validity and reproducibility ${ }^{45}$. In addition, the current finding between coffee intake and amyloid was significant even after controlling the effect of clinical diagnosis on cognitive status, and the reported frequency of coffee intake was not related with the proportion of MCI (Table 1). Finally, it is unclear which ingredient(s) in coffee acts on $A \beta$ pathology. Although caffeine is among hundreds of bioactive compounds in coffee $^{46}$, it is the most widely studied ingredient against $\mathrm{A} \beta$ pathology ${ }^{16-18}$. Other bioactive compounds include chlorogenic acid, polyphenols, small amount of minerals, and vitamin $B_{3}$, which have also been investigated ${ }^{47-49}$. However, it remains controversial whether a single ingredient in coffee is effective against $A \beta$ pathology or whether a combination of ingredients is effective. 
Therefore, further investigations are needed to clarify which ingredient(s) in coffee are important for reducing $\mathrm{A} \beta$ pathology. The comparison between coffee with and without caffeine may give us a clue on the specific effect of caffeine.

In conclusion, the findings of present study suggest that higher lifetime coffee intake is likely to contribute to lowering the risk of $\mathrm{AD}$ or related cognitive decline by reducing pathological cerebral amyloid deposition.

\section{Acknowledgements}

We thank the KBASE study participants and their caregivers. This study was supported by a grant from the Ministry of Science, ICT, and Future Planning, Republic of Korea (Grant no. NRF-2014M3C7A1046042) and a grant of the Korea Health Technology R\&D Project through the Korea Health Industry Development Institute (KHIDI), funded by the Ministry of Health \& Welfare, Republic of Korea (Grant nos. HI18C0630 and HI19C0149). The funding source had no role in the study design, data collection, data analysis, data interpretation, writing of the paper, or decision to submit it for publication.

\section{Author details}

'Department of Neuropsychiatry, Hallym University Dongtan Sacred Heart Hospital, 7 Keunjaebong-Gil, Hwaseong, Gyeonggi 18450, Republic of Korea. ${ }^{2}$ Department of Psychiatry, Hallym University College of Medicine, Chuncheon, Gangwon 24252, Republic of Korea. ${ }^{3}$ Institute of Human Behavioral Medicine, Medical Research Center Seoul National University, Seoul 03080, Republic of Korea. ${ }^{4}$ Department of Neuropsychiatry, Seoul National University Hospital, Seoul 03080, Republic of Korea. ${ }^{5}$ Department of Psychiatry, Sanggye Paik Hospital, Inje University College of Medicine, Seoul 01757, Republic of Korea. ${ }^{6}$ Department of Neuropsychiatry, SMG-SNU Boramae Medical Center, Seoul 07061, Republic of Korea. ${ }^{7}$ Department of Psychiatry, Seoul National University College of Medicine, Seoul 03080, Republic of Korea. ${ }^{8}$ Department of Nuclear Medicine, SMG-SNU Boramae Medical Center, Seoul 07061, Republic of Korea. ${ }^{9}$ Department of Radiology, Seoul National University College of Medicine, Seoul 03080, Republic of Korea

\section{Authors' contributions}

J.W.K. and D.Y.L. conceived and designed the study. M.S.B., D.Y., J.H.L. S.Y.J., G.J., H.N., B.K.S., J.Y.L., Y.K.K., S.A.S., C.-H.S. and D.Y.L. were involved in acquisition, or analysis and interpretation of the data and helped to draft the paper. J.W.K., M. S.B., D.Y., J.H.L. and D.Y.L. were major contributors in writing the paer and critically revising the paper for intellectual content. D.Y.L. served as principal investigator and supervised the study. All authors read and approved the final paper.

\section{Availability of data and materials}

The datasets generated and analyzed during the present study are not publicly available, owing to ethics considerations and privacy restrictions. Data may be obtained from the corresponding author after approval by the Institutional Review Board of the Seoul National University Hospital, South Korea has been sought.

\section{Ethics approval and consent to participate}

The study protocol was approved by the Institutional Review Boards of Seoul National University Hospital and SNU-SMG Boramae Center, Seoul, South Korea, and the study was conducted in accordance with the recommendations of the current version of the Declaration of Helsinki. All subjects provided written informed consent.

\section{Conflict of interest}

The authors declare that they have no conflict of interest.

\section{Publisher's note}

Springer Nature remains neutral with regard to jurisdictional claims in published maps and institutional affiliations.
Supplementary Information accompanies this paper at (https://doi.org/ 10.1038/s41398-019-0604-5).

Received: 25 March 2019 Revised: 22 June 2019 Accepted: 30 July 2019 Published online: 22 October 2019

\section{References}

1. Loftfield, E. et al. Coffee drinking is widespread in the United States, but usual intake varies by key demographic and lifestyle factors. J. Nutr. 146, 1762-1768 (2016).

2. Spiller, M. A. The chemical components of coffee. Prog. Clin. Biol. Res. 158, 91-147 (1984).

3. Larsson, S. C., Virtamo, J. \& Wolk, A. Coffee consumption and risk of stroke in women. Stroke 42, 908-912 (2011).

4. Mostofsky, E., Rice, M. S., Levitan, E. B. \& Mittleman, M. A. Habitual coffee consumption and risk of heart failure: a dose-response meta-analysis. Circ. Heart Fail. 5, 401-405 (2012).

5. Wang, A. et al. Coffee and cancer risk: a meta-analysis of prospective observational studies. Sci. Rep. 6, 33711 (2016).

6. Akash, M. S., Rehman, K. \& Chen, S. Effects of coffee on type 2 diabetes mellitus. Nutrition 30, 755-763 (2014).

7. Lucas, M. et al. Coffee, caffeine, and risk of completed suicide: results from three prospective cohorts of American adults. World J. Biol. Psychiatry 15, 377-386 (2014).

8. Ross, G. W. et al. Association of coffee and caffeine intake with the risk of Parkinson disease. J. Am. Med. Assoc. 283, 2674-2679 (2000).

9. Ding, M. et al. Association of Coffee Consumption With Total and CauseSpecific Mortality in 3 Large Prospective Cohorts. Circulation 132, 2305-2315 (2015).

10. Eskelinen, M. H. \& Kivipelto, M. Caffeine as a protective factor in dementia and Alzheimer's disease. J. Alzheimer Dis. 20(Suppl 1), S167-S174 (2010).

11. Lindsay, J. et al. Risk factors for Alzheimer's disease: a prospective analysis from the Canadian Study of Health and Aging. Am. J. Epidemiol. 156, 445-453 (2002).

12. Eskelinen, M. H., Ngandu, T., Tuomilehto, J., Soininen, H. \& Kivipelto, M. Midlife coffee and tea drinking and the risk of late-life dementia: a population-based CAIDE study. J. Alzheimer Dis. 16, 85-91 (2009).

13. Arab, L. et al. Gender differences in tea, coffee, and cognitive decline in the elderly: the Cardiovascular Health Study. J. Alzheimer Dis. 27, 553-566 (2011).

14. van Gelder, B. M. et al. Coffee consumption is inversely associated with cognitive decline in elderly European men: the FINE Study. Eur. J. Clin. Nutr. 61, 226-232 (2007).

15. Vercambre, M. N., Berr, C., Ritchie, K. \& Kang, J. H. Caffeine and cognitive decline in elderly women at high vascular risk. J. Alzheimer Dis. 35, 413-421 (2013).

16. Arendash, G. W. et al. Caffeine reverses cognitive impairment and decreases brain amyloid-beta levels in aged Alzheimer's disease mice. J. Alzheimer Dis. 17, 661-680 (2009).

17. Cao, C. et al. Caffeine suppresses amyloid-beta levels in plasma and brain of Alzheimer's disease transgenic mice. J. Alzheimer Dis. 17, 681-697 (2009).

18. Arendash, G. W. et al. Caffeine protects Alzheimer's mice against cognitive impairment and reduces brain beta-amyloid production. Neuroscience $\mathbf{1 4 2}$, 941-952 (2006).

19. Byun, M. S. et al. Korean Brain Aging Study for the Early Diagnosis and Prediction of Alzheimer's Disease: methodology and baseline sample characteristics. Psychiatry Investig. 14, 851-863 (2017)

20. Morris, J. C. The Clinical Dementia Rating (CDR): current version and scoring rules. Neurology 43, 2412-2414 (1993).

21. Lee, D. Y. et al. A normative study of the CERAD neuropsychological assessment battery in the Korean elderly. J. Int. Neuropsychological Soc. 10, 72-81 (2004).

22. Lee, J. H. et al. Development of the Korean version of the Consortium to Establish a Registry for Alzheimer's Disease Assessment Packet (CERAD-K): clinical and neuropsychological assessment batteries. J. Gerontol. Ser. B Psychol. Sci. Soc. Sci. 57, P47-P53 (2002).

23. Driscoll, I. et al. Relationships between caffeine intake and risk for probable dementia or global cognitive impairment: the Women's Health Initiative Memory Study. J. Gerontol. Ser. A Biol. Sci. Med. Sci. 71, 1596-1602 (2016). 
24. Wilson, R. S. et al. Early and late life cognitive activity and cognitive systems in old age. J. Int. Neuropsychol. Soc. 11, 400-407 (2005).

25. Wilson, R. S., Scherr, P. A., Schneider, J. A., Tang, Y. \& Bennett, D. A. Relation of cognitive activity to risk of developing Alzheimer disease. Neurology 69, 1911-1920 (2007).

26. Ko, K. et al. Early-life cognitive activity is related to reduced neurodegeneration in Alzheimer signature regions in late life. Front. Aging Neurosci. 10, 70 (2018).

27. DeCarli, C. et al. Memory impairment, but not cerebrovascular disease, predicts progression of MCl to dementia. Neurology 63, 220-227 (2004).

28. Kim, J. Y. et al. Standardization of the korean version of the geriatric depression scale: reliability, validity, and factor structure. Psychiatry Investig. 5, 232-238 (2008).

29. Wenham, P. R., Price, W. H. \& Blandell, G. Apolipoprotein E genotyping by onestage PCR. Lancet 337, 1158-1159 (1991)

30. Park, J. C. et al. Plasma tau/amyloid-beta1-42 ratio predicts brain tau deposition and neurodegeneration in Alzheimer's disease. Brain 142, 771-786 (2019).

31. Reiman, E. M. et al. Fibrillar amyloid-beta burden in cognitively normal people at 3 levels of genetic risk for Alzheimer's disease. Proc. Natl Acad. Sci. USA 106, 6820-6825 (2009).

32. Jack, C. R. et al. Age-specific population frequencies of cerebral betaamyloidosis and neurodegeneration among people with normal cognitive function aged 50-89 years: a cross-sectional study. Lancet Neurol. 13 997-1005 (2014).

33. Klunk, W. E. Amyloid imaging as a biomarker for cerebral beta-amyloidosis and risk prediction for Alzheimer dementia. Neurobiol. Aging 32(Suppl 1), S20-\$36 (2011).

34. Gottesman, R. F. et al. Association between midlife vascular risk factors and estimated brain amyloid deposition. J. Am. Med. Assoc. 317, 1443-1450 (2017).

35. Tsai, J. Z. et al. Automated segmentation and quantification of white matter hyperintensities in acute ischemic stroke patients with cerebral infarction. PloS ONE 9, e104011 (2014).

36. Moon, S. W. et al. The ankle-brachial index is associated with cerebral betaamyloid deposition in cognitively normal older adults. J. Gerontol. Ser. A Biol. Sci. Med. Sci. 74, 1141-1148 (2019).
37. Li, S. et al. Caffeine, through adenosine a3 receptor-mediated actions, suppresses amyloid-beta protein precursor internalization and amyloid-beta generation. J. Alzheimer Dis. 47, 73-83 (2015).

38. Cao, C. et al. Caffeine synergizes with another coffee component to increase plasma GCSF: linkage to cognitive benefits in Alzheimer's mice. J. Alzheimer Dis. 25, 323-335 (2011).

39. Gelber, R. P., Petrovitch, H., Masaki, K. H., Ross, G. W. \& White, L. R. Coffee intake in midlife and risk of dementia and its neuropathologic correlates. J. Alzheimer Dis. 23, 607-615 (2011).

40. Grobbee, D. E. et al. Coffee, caffeine, and cardiovascular disease in men. N. Engl. J. Med. 323, 1026-1032 (1990).

41. Mostofsky, E., Schlaug, G., Mukamal, K. J., Rosamond, W. D. \& Mittleman, M. A. Coffee and acute ischemic stroke onset: the Stroke Onset Study. Neurology 75, 1583-1588 (2010).

42. Ritchie, $\mathrm{K}$. et al. Caffeine, cognitive functioning, and white matter lesions in the elderly: establishing causality from epidemiological evidence. J. Alzheimer Dis. 20(Suppl 1), S161-S166 (2010).

43. Jack, C. R. et al. Hypothetical model of dynamic biomarkers of the Alzheimer's pathological cascade. Lancet Neurol. 9, 119-128 (2010).

44. Jack, C. R. et al. Tracking pathophysiological processes in Alzheimer's disease: an updated hypothetical model of dynamic biomarkers. Lancet Neurol. 12, 207-216 (2013).

45. Watson, E. J., Kohler, M., Banks, S. \& Coates, A. M. Validation and reproducibility of an Australian caffeine food frequency questionnaire. Int J. Food Sci. Nutr. $\mathbf{6 8}$ 617-626 (2017).

46. Nuhu, A. A. Bioactive micronutrients in coffee: recent analytical approaches for characterization and quantification. ISRN Nutr. 2014, 384230 (2014).

47. Fukuyama K. et al. Roasted coffee reduces beta-amyloid production by increasing proteasomal beta-secretase degradation in human neuroblastoma SH-SY5Y Cells. Mol. Nutr. Food Res. 62, e1800238 (2018).

48. Dhouafli Z. et al. Inhibition of protein misfolding and aggregation by natural phenolic compounds. Cell Mol. Life Sci. 75, 3521-3538 (2018).

49. Turunc Bayrakdar, E., Uyanikgil, Y., Kanit, L., Koylu, E. \& Yalcin, A. Nicotinamide treatment reduces the levels of oxidative stress, apoptosis, and PARP-1 activity in Abeta(1-42)-induced rat model of Alzheimer's disease. Free Radic. Res. 48, 146-158 (2014). 